\title{
Towards autotrophic tissue engineering: Photosynthetic gene therapy for regeneration
}

\author{
Myra Noemi Chávez a, b, Thilo Ludwig Schenck ${ }^{\mathrm{a}, \mathrm{j}}$, Ursula Hopfner a, \\ Carolina Centeno-Cerdas ${ }^{\text {a, }}$, Ian Somlai-Schweiger ${ }^{\mathrm{d}}$, Christian Schwarz ${ }^{\mathrm{e}}$,

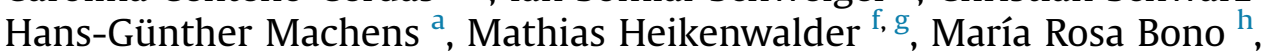 \\ Miguel L. Allende ${ }^{b}$, Jörg Nickelsen ${ }^{e}$, José Tomás Egaña ${ }^{a, b, i, *}$ \\ ${ }^{a}$ Department of Plastic Surgery and Hand Surgery, University Hospital rechts der Isar, Technische Universität München, Germany \\ ${ }^{\mathrm{b}}$ FONDAP Center for Genome Regulation, Faculty of Science, Universidad de Chile, Santiago, Chile \\ ${ }^{\mathrm{c}}$ Biotechnology Research Center, Costa Rica Institute of Technology, Cartago, Costa Rica \\ d Department of Nuclear Medicine, University Hospital rechts der Isar, Technische Universität, München, Germany \\ e Molekulare Pflanzenwissenschaften, Biozentrum Ludwig-Maximilians-Universität München, Planegg-Martinsried, Germany \\ ${ }^{\mathrm{f}}$ Institute of Virology, Technische Universität München, Germany \\ ${ }^{g}$ Division of Chronic Inflammation and Cancer, German Cancer Research Center (DKFZ), Heidelberg, Germany \\ hepartment of Biology, Faculty of Science, Universidad de Chile, Santiago, Chile \\ ${ }^{\mathrm{i}}$ Institute for Medical and Biological Engineering, Schools of Engineering, Biological Sciences and Medicine, Pontificia Universidad Católica de Chile, \\ Santiago, Chile \\ j Handchirurgie, Plastische Chirurgie, Ästhetische Chirurgie der Ludwig-Maximilians Universität München, Germany
}

\section{A R T I C L E I N F O}

\section{Article history:}

Received 31 July 2015

Received in revised form

30 September 2015

Accepted 5 October 2015

Available online 8 October 2015

\section{Keywords:}

Regenerative medicine

Drug delivery

Molecular therapy

Biomaterials

Algae biotechnology

Oxygen

Hypoxia

\begin{abstract}
A B S T R A C T
The use of artificial tissues in regenerative medicine is limited due to hypoxia. As a strategy to overcome this drawback, we have shown that photosynthetic biomaterials can produce and provide oxygen independently of blood perfusion by generating chimeric animal-plant tissues during dermal regeneration. In this work, we demonstrate the safety and efficacy of photosynthetic biomaterials in vivo after engraftment in a fully immunocompetent mouse skin defect model. Further, we show that it is also possible to genetically engineer such photosynthetic scaffolds to deliver other key molecules in addition to oxygen. As a proof-of-concept, biomaterials were loaded with gene modified microalgae expressing the angiogenic recombinant protein VEGF. Survival of the algae, growth factor delivery and regenerative potential were evaluated in vitro and in vivo. This work proposes the use of photosynthetic gene therapy in regenerative medicine and provides scientific evidence for the use of engineered microalgae as an alternative to deliver recombinant molecules for gene therapy.
\end{abstract}

๑) 2015 Elsevier Ltd. All rights reserved.

\section{Introduction}

Oxygen is an essential molecule for cell metabolism. It plays a key role in tissue survival and regeneration, yet paradoxically, its appropriate delivery is one of the major problems for the clinical translation of non-vascularized tissue engineering approaches [1]. As a result, cells within bioartificial tissue constructs are stringently

\footnotetext{
* Corresponding author. Dept. Plastic Surgery and Hand Surgery, University Hospital rechts der Isar, Technische Universität München, Ismaningerstr. 22, 81675 Munich, Germany.

E-mail address: tomasega@gmail.com (J.T. Egaña).
}

dependent on oxygen diffusion, which barely reaches few hundred micrometers and limits the construction of artificial tissues to nonclinically relevant sizes [2]. Besides the basic oxygen requirements, tissue regeneration relies on several bioactive molecules, such as growth factors, that control key processes including inflammation, angiogenesis and tissue remodeling [3-5]. Scaffold bioactivation with growth factors has been intensively investigated as strategy to enhance the regenerative potential of engineered tissues [6]. However, direct growth factor administration has major limitations related to the short biological half-life of the molecules, and the consequent need for repeated administration of large and potentially toxic bulk doses $[7,8]$. Conversely, traditional gene therapy 
represents a better strategy to provide a constant growth factor supply. Yet, this approach raises ethical and technical concerns that affect their translation into clinical settings. For instance, the delivery of target genes often creates risk of oncogenicity, insertional mutagenesis and viral vector-related immune reactions [9,10], while target tissue-specificity and high transduction efficiency are still unrealized for non-viral gene delivery methods [11,12]. Additionally, sustained transgene expression is not guaranteed if the transgenic cell survival depends of local oxygen tension at the injection site.

Recently, we have introduced HULK (from the German Hyperoxie Unter Licht Konditionierung) as a novel concept to deliver oxygen into biomaterials independently of blood vessel perfusion. The main idea behind the HULK approach is that, by incorporating photosynthetic microalgae such as Chlamydomonas reinhardtii (C. reinhardtii) into artificial constructs, the local induction of photosynthesis could be able to supply the metabolic needs of bioengineered tissues in vitro [13] and in vivo [14]. To take HULK one step forward, in this work we evaluated the feasibility of implanting photosynthetic scaffolds in fully immunocompetent mice. Then, we explored the idea of using genetically modified microalgae to engineer photosynthetic scaffolds that, in addition to oxygen, could provide other pro-regenerative molecules to the wounded tissue. As a proof of concept, we created a gene modified C. reinhardtii strain that constitutively secretes the human vascular endothelial growth factor VEGF-165 (VEGF).

In this work we propose that the activation of biomaterials with gene modified microalgae could be used to locally deliver oxygen and other pro-regenerative molecules into bioartificial tissue constructs.

\section{Materials and methods}

\subsection{Cell culture of $C$. reinhardtii}

The cell-wall deficient, cw15-30-derived UVM4 C. reinhardtii strain [15] was grown photomixotrophically at $20^{\circ} \mathrm{C}$ on either solid Tris Acetate Phosphate (TAP) medium or in liquid TAPS-medium supplemented with $1 \%(\mathrm{w} / \mathrm{v})$ sorbitol [16]. For light stimulation, a lamp with the full spectrum of white light (Nano Light, 11 Watt, Dennerle, Vinningen, Germany) was used to provide constant illumination ( 2500 lux, eq. $72.5 \mu \mathrm{E} / \mathrm{m}^{2} \cdot \mathrm{s}^{1}$ ). Cell concentration in the culture was determined using a Casy Counter TT (Roche Diagnostics, Mannheim, Germany).

\subsection{Cell seeding in the scaffolds}

For in vitro experiments, if not stated otherwise, Integra ${ }^{\circledR}$ matrix single layer (Integra Life Science Corporation, Plainsboro, NJ, USA) was used as a scaffold. For seeding, a C. reinhardtii, suspension was mixed with fibrinogen (Tissucol-Kit 2.0 Immuno, Baxter $\mathrm{GmbH}$, Unterschleißheim, Germany) in a 1:1 ratio to the respective final concentration. Pieces of IM (ø $12 \mathrm{~mm}, 1 \mathrm{~mm}$ thick) were cut using a biopsy punch and dried with sterile gauze. $50 \mu \mathrm{l}$ thrombin-solution (Tissucol-Kit 2.0 Immuno, Baxter GmbH, Unterschleißheim, Germany) followed by $100 \mu \mathrm{l}$ of the algae-fibrinogen solution were pipetted over each scaffold. Control scaffolds were prepared by adding $50 \mu \mathrm{l}$ thrombin and $100 \mu \mathrm{l}$ TAPS-fibrinogen 1:1 solution. After $1 \mathrm{~h}$, the scaffolds were covered with TAPS-buffer and incubated over the desired period of time at room temperature and constant illumination. Pictures of the scaffolds at different time points were taken with a stereomicroscope (Stereomicroscope Stemi 2000-C, Carl Zeiss AG, Oberkochen, Germany) or an inverted phase-contrast microscope (Axiovert 25, Carl Zeiss AG, Oberkochen, Germany) and analyzed with the Axiovision software (Carl
Zeiss AG, Oberkochen, Germany).

\subsection{Construction of transformation vector}

The coding sequence for the human vascular endothelial growth factor A isoform 1 (accession number NP_001165097) was adapted to the codon bias of $C$. reinhardtii. The synthetic gene $\mathrm{pBC} 1$ VEGF165 was assembled from synthetic oligonucleotides and PCR-products. The sequence was inserted into an expression cassette derived from the endogenous PsaD gene $5^{\prime}$ and $3^{\prime}$ UTRs regulatory elements and behind the export sequence encoding the 21 amino acid leader peptide of the $C$. reinhardtii extracellular enzyme arylsulfatase (ARS2) to target the transgenic proteins for secretion into the culture medium. The pBC1-CrGFP_J131 basis vector [15] contained the APHVIII resistance gene for selection on paromomycin, whose expression is controlled by the constitutively active HSP70/RBCS2 promoter regions and the first intron of the RBCS2 gene. The transgene fragment was cloned into the vector backbone pBC1-CrGFP_J131 using NdeI and EcoRI cloning sites. The final constructs were verified by sequencing. The plasmid DNA was replicated and purified from transformed $E$. coli $\mathrm{K} 12$ (dam $+\mathrm{dcm}+$ tonA rec-) bacteria. All this was performed by Gene Art AG, Life Technologies, Regensburg, Germany.

\subsection{Transformation of $C$. reinhardtii}

$1 \cdot 10^{7}$ UVM4 C. reinhardtii-cells were suspended to a volume of $300 \mu \mathrm{l}$ and vortexed with $\varnothing 0.5 \mathrm{~mm}$ glass beads for $20 \mathrm{~s}$ in the presence of $5 \mu \mathrm{g}$ plasmid DNA. Then, the transformants were seeded in TAPS-liquid medium and incubated overnight protected from light under continuous shaking. Next, the algae were seeded over TAP-Agar plates containing $10 \mu \mathrm{g} / \mathrm{ml}$ paromomycin and incubated for the first three days under weak illumination. Upon successful transformation, the new created strains integrated an antibiotic-resistance gene and were therefore able to grow selectively in the presence of paromomycin. The plates were then moved to conditions with standard light exposition (2500 lux), until the colonies were large enough to be picked and platted into a fresh plate. Clones were subsequently maintained in solid TAP-medium under selective conditions.

\subsection{Southern blot}

Genomic DNA from $C$. reinhardtii cells was extracted using the DNeasy Plant Mini Kit (Qiagen N.V., Lumburg, Netherlands) according to the manufacturer's instructions. $10 \mu \mathrm{g}$ DNA were digested using the restriction enzymes HindIII and BamHI at $37{ }^{\circ} \mathrm{C}$ for 48 h. Samples were separated by $0.8 \%$ agarose-TBE gel electrophoresis for $16 \mathrm{~h}$ at $25 \mathrm{~V}$. Following depurination ( $15 \mathrm{~min}, 0.25 \mathrm{M}$ $\mathrm{HCl}$ ), denaturation (30 $\mathrm{min}, 0.4 \mathrm{M} \mathrm{NaOH}, 0.6 \mathrm{M} \mathrm{NaCl}$ ) and neutralization (30 min, $1 \mathrm{M}$ Tris- $\mathrm{HCl} \mathrm{pH} 8.0,1.5 \mathrm{M} \mathrm{NaCl}$ ) genomic DNA was transferred to a nylon membrane (Roti-Nylon plus,; CarlRoth, Karlsruhe, Germany) in $20 \times$ SCC ( $3 \mathrm{M} \mathrm{NaCl}, 0.3 \mathrm{M}$ Trisodiumcitrate) overnight. Digoxigenin-nucleotide labeled DNAprobes (Digoxigenin-11-dUTP alkali-labile, Roche, Basel, Switzerland) were obtained using the same primer pairs and conditions as for the PCR (see below). After blocking unspecific binding sites, immobilized genomic DNA was incubated with the labeled probes for $14 \mathrm{~h}$ at $68{ }^{\circ} \mathrm{C}$ for hybridization. Signals were detected using an alkaline phosphatase conjugated anti-DIG antibody (Roche, Basel, Switzerland) and CDP* (Roche, Basel, Switzerland) as reaction substrate. 


\subsection{PCR-assay}

The integration of the recombinant gene was confirmed by PCR using the gene specific primer pair $5^{\prime}$-GAAGTTCATGGACGTGTACC$3^{\prime}$ and $5^{\prime}$-TTGTTGTGCTGCAGGAAG-3', 258 bp product. The primer sequences for the amplification of the $p s b D$ sequence were $5^{\prime}$ GCCGTAGGGTTGAATG-3' and 5'-GTTGGTGTCAACTTGGTGG-3', 413 bp product (Metabion $\mathrm{GmbH}$, Planegg/Steinkirchen, Germany).

\subsection{Western blot}

Total soluble protein extracts were prepared by resuspending $5 \cdot 10^{7}$ pelleted $C$. reinhardtii cells ( $5 \mathrm{~min}, 10,000 \mathrm{rpm}$ ) in $100 \mu \mathrm{l}$ lysis buffer (100 mM Tris-HCL, 10 mM EDTA, 0.5\% Triton-X-100, $25 \mu \mathrm{g} / \mathrm{ml}$ pepstatin, $25 \mu \mathrm{g} / \mathrm{ml}$ leupeptin, $25 \mu \mathrm{g} / \mathrm{ml}$ aprotinin). Cells were disrupted and lysates were homogenized by pipetting up and down through a $1 \mathrm{ml}$ syringe and stored at $-80{ }^{\circ} \mathrm{C}$ for further analysis. Equal protein amounts were denatured at $95{ }^{\circ} \mathrm{C}$ for $5 \mathrm{~min}$ in the presence of reducing loading buffer ( $0.03 \mathrm{M}$ Tris $\mathrm{pH} 6.8,10 \% \mathrm{SDS}$, 15\% Glycerin, 0.03\% bromphenolblue, 12.5\% 2-mercaptoethanol), separated by gel-electrophoresis and transferred to PVDFmembranes. Commercially available human VEGF-165 was loaded as a positive control (Preprotech, NJ, USA). Protein detection was performed using a monoclonal rabbit anti-VEGF primary antibody (1:1000 dilution, ab51917, Abcam, plc, Cambridge, UK) and rabbit anti-HSP70 serum (kindly provided by Michael Schroda, Department of Molecular Biotechnology and Systems Biology, TUK, Kaiserslautern, Germany), and a goat anti-rabbit HRP secondary antibody (1:5000, Dianova GmbH, Hamburg, Germany), using the SuperSignal West Pico detection system (Pierce-Thermo Fisher Scientific Inc, IL, USA).

\subsection{Enzyme-linked ImmunoSorbent assay (ELISA)}

$5 \cdot 10^{7}$ C. reinhardtii cells were seeded in triplicate in $10 \mathrm{ml}$ volume and incubated at room temperature for 4 days under constant agitation (150 rpm) and illumination (2500 lux). Culture supernatants were collected and then stored at $-80{ }^{\circ} \mathrm{C}$ until their analysis. Protein lysates from the explanted scaffolds were obtained by mechanical disruption in RIPA buffer with proteinase inhibitors (PIC; BD Pharmingen, NJ, USA), Pefabloc SC-Protease Inhibitor (CarlRoth, Karlsruhe, Germany), cOmplete (Roche, Basel, Switzerland), phenylmethylsulfonyl fluoride (PMSF; Sigma-Aldrich, MO, USA). To assess the secretion of the recombinant proteins from the encapsulated algae, scaffolds were seeded as described before with transgenic algae at a final concentration of $0.5 \cdot 10^{6}$ cells per scaffold and placed in 12 well-plates with $2 \mathrm{ml}$ TAPS-medium. Medium was changed every day and collected every two days. For quantification of the secreted recombinant proteins in the supernatant samples and lysates, the human VEGF Quantikine ELISA kit was used according to the manufacturer's instructions. Here, a monoclonal antibody raised against the Sf 21-expressed recombinant human protein VEGF-165 was used as a capture antibody and a specific polyclonal antibody as the detection antibody.

\subsection{Recovery of the recombinant protein from the culture supernatant}

The supernatants from confluent $C$. reinhardtii cultures were passed first through a $0.22 \mu \mathrm{m}$ filter. Then using a filtration tube capable of retaining peptides above 3000 Da (Vivaspin 15R Hydrosart, Sarstedt, Göttingen, Germany) VEGF-165 was recovered by centrifugation $(3000 \times \mathrm{g}, 47 \mathrm{~min})$. In a final filtration step, the diluent medium was changed to cell starvation medium (RPMI 1640 , with stable glutamine and $2.0 \mathrm{~g} / \mathrm{L}$ NaHCO3; Biochrom, Berlin,
Germany) supplemented with $1 \%$ fetal calf serum (FCS-Gold, PAA, Pasching, Austria) and $1 \%$ antibiotic/antimycotic $(100 \times \mathrm{ab} / \mathrm{am}$; Capricorn Scientific, Ebsdorfergrund, Germany).

\subsection{Cell culture of human endothelial progenitor cells}

Human umbilical vein endothelial cells (HUVECs) were either purchased (Promocell, Heidelberg, Germany) or obtained from umbilical cord donors as described before [17]. Cells were maintained in supplemented Endothelial Cell Growth Medium 2 (Promocell, Heidelberg, Germany) with $1 \%$ penicillin/streptomycin (Biochrom, Berlin, Germany) under standard cell culture conditions $\left(37{ }^{\circ} \mathrm{C}, 5 \% \mathrm{CO}_{2}\right.$ ). In all experimental settings, cells from passages 2-4 were used.

\subsection{Receptor phosphorylation assay}

HUVECs $\left(1 \cdot 10^{5}\right.$ cells per well) were seeded on a 12 well-plate, cultured for $24 \mathrm{~h}$ and then starved for $16 \mathrm{~h}$ before activation. Cells were then stimulated for $2 \mathrm{~min}$, with either $50 \mathrm{ng} / \mathrm{ml}$ recombinant VEGF-165 (Preprotech, NJ, USA), or the concentrated protein supernatants of $30 \mathrm{ml}$ confluent cultures of the genetic modified and wild-type strains (approx. $2 \cdot 10^{7}$ cells $/ \mathrm{ml}$ ). Cells were then snap-frozen by submerging the plate in liquid nitrogen and lysed in RIPA-buffer with proteinase inhibitors and phosphatase inhibitors (Phosphatase Inhibitor Mini Tablets; Pierce-Thermo Fisher Scientific Inc, IL, USA). Cells were scratched from the wellfloor and lysates were homogenized by pipetting up and down through a $1 \mathrm{ml}$ syringe, centrifuged for $5 \mathrm{~min}$ at $9300 \mathrm{~g}$ and stored at $-80{ }^{\circ} \mathrm{C}$ for further analysis. Equal protein amounts were separated by gel-electrophoresis under reducing conditions and then blotted to PVDF-membranes (Bio-Rad Laboratories, Hercules, California, USA). The mononuclear antibodies rabbit mAb anti-VEGFR-2 and rabbit $m A b$ anti-phospho-VEGFR-2 (Tyr1175) (CellSignalling, MA, USA) were used for the detection of the phosphorylated and non-phosphorylated receptor epitope with overnight incubation periods. Pixel intensity quantification was performed with the software Fusion (Peqlab, Erlangen, Germany).

\subsection{Cell proliferation assay}

HUVECs $\left(2 \cdot 10^{4}\right.$ cells per well) were seeded in a 24 -well plate. Cells were grown into $60 \%$ confluence. Next, medium was replaced by starvation medium overnight. Cells were then stimulated every $12 \mathrm{~h}$ for $48 \mathrm{~h}$ with either $50 \mathrm{ng} / \mathrm{ml}$ recombinant VEGF-165, the concentrated protein supernatants of confluent cultures of the genetically modified algae or wild-type strains. Then, cells were trypsinized (Trypsin/EDTA $1 \times$, PAA, GE Healthcare, USA) and counted using trypan blue (Trypan Blue solution; Sigma-Aldrich, MO, USA) as dead-staining. For immunohistological analysis, cells were fixed in 3.5-3.7\% formaldehyde (Otto Fischar GmbH und Co. KG, Saarbrücken, Germany) by the end of the experimental time and stained with a monoclonal rabbit anti-VEGF primary antibody (1:1000, ab51917, Abcam, plc, Cambridge, UK) and a goat anti rabbit secondary antibody (1:1000, Alexa 568, A11011,Thermo Fisher Scientific, Waltham, USA).

\subsection{Cell migration assay}

HUVECs $\left(1 \cdot 10^{4}\right.$ cells per well) were seeded in $70 \mu \mathrm{l}$ culture medium into each well of a culture insert (Ibidi, Martinsried, Germany). Cells were grown into confluence. Next, culture inserts were removed and medium was replaced by starvation medium overnight. Cells were then stimulated every for $24 \mathrm{~h}$ with either $50 \mathrm{ng} /$ $\mathrm{ml}$ recombinant VEGF-165 (positive control), the concentrated 
protein supernatants of confluent cultures of the genetically modified strain or the wild-type strain. Then, cells were stained with calcein AM (Invitrogen, Oregon, USA), according to manufacturer's instructions, to visualize living cells. Microscopic pictures were taken with an Axio Observer Z1 microscope (Zeiss, Göttingen, Germany) and the number of migrating cells into the open wound area was quantified with the software Image [18].

\subsection{Zebrafish breeding}

Zebrafish (Danio rerio) embryos were obtained from the laboratory-breeding colony. Embryos were collected by natural spawning and raised at $28.5^{\circ} \mathrm{C}$ in $\mathrm{E} 3$ medium $(5 \mathrm{mM} \mathrm{NaCl}, 0.17 \mathrm{mM}$ $\mathrm{KCl}, 0.33 \mathrm{mM} \mathrm{CaCl}_{2}, 0.3 \mathrm{mM} \mathrm{MgSO} 4$ equilibrated to $\mathrm{pH} 7.0$ ); egg water was changed daily. Embryonic ages are expressed as days post-fertilization (dpf). Animals were anesthetized with MS-222 (Tricaine, A5040, Sigma-Aldrich, MO, USA) before each experiment. All procedures complied with guidelines of the Animal Ethics Committee of the University of Chile.

\subsection{Zebrafish model of angiogenesis}

Zebrafish ( $D$. rerio) embryos of the transgenic strain $\mathrm{Tg}$ (fli1a:EGFP)y1 [19] were collected and raised at $28.5{ }^{\circ} \mathrm{C}$ in E3 medium. C. reinhardtii cells were brought to a concentration of $5 \cdot 10^{7}$ cells/ml in fresh TAPS-medium and loaded to glass capillary needles. Previously selected and dechorionated transgenic zebrafish embryos of $1 \mathrm{dpf}$ were injected with approximately $10 \mathrm{nl}$ of the respective cell suspension into the yolk sack. 20-30 zebrafish larvae were injected per group. Embryos were incubated for $24 \mathrm{~h}$ in E3 medium and for further $48 \mathrm{~h}$ in E3 with Phenylthiourea (PTU, NPhenylthiourea, Sigma-Aldrich, MO, USA) at $28^{\circ} \mathrm{C}$ and imaged as described below in this section.

\subsection{Confocal microscopy, fluorescence microscopy and image processing}

Living zebrafish embryos were chosen randomly, anesthetized and mounted in $0.75 \%$ low-melting point agarose containing $5 \%$ Tricaine in a $35 \mathrm{~mm}$ imaging dish, and fixed to a lateral position. Embryos were first examined using an epifluorescence-inverted microscope (Olympus scan, Olympus Biosystems, Munich, Germany). Z-stack images were taken at a fixed $15 \mu \mathrm{m}$ intervals in a confocal microscope (Zeiss LSM 510 Meta, Carl Zeiss AG, Oberkochen, Germany). Z-Projections of the stacks were then merged and combined to generate a mosaic image of the whole fish using the software Zeiss LSM Image Browser Version 3.1.0.99, Image $1.46 \mathrm{r}$ (Java 1.6.0_20 (64-bit)), the software ImageJ [18] and the plugin Mosaicj [20].

\subsection{Chlorophyll measurements}

Scaffolds were seeded with $3 \cdot 10^{5} \mathrm{C}$. reinhardtii cells as described in section 2.2. At different culture times (days $1,7,14$ ) the scaffolds were mechanically disrupted and optical density was measured at $435 \mathrm{~nm}$ (Nanodrop, Thermo Scientific, Waltham, MA, USA) as described before [13].

\subsection{Oxygen release measurements}

Scaffolds were seeded at a final concentration of $2.5 \cdot 10^{6}$ cells per scaffold and incubated for 5 days at room temperature, followed by a $16 \mathrm{~h}$ dark time incubation period, prior to the start of the measurement. Afterwards, samples were placed under hypoxic conditions $\left(1 \% \mathrm{pO}_{2}\right)$ and oxygen concentrations in vitro were constantly measured by the SensorDish ${ }^{\circledR}$ Reader (PreSens GmbH, Regensburg, Germany) in Oxodish ${ }^{\circledR}$ well-plates according to the manufacturer's instructions. For light stimulation, a lamp (Nano Light, 11 Watt, Dennerle, Vinningen, Germany) was placed at $40 \mathrm{~cm}$ distance over the sample. Oxygen concentration was recorded over $24 \mathrm{~h}$.

\subsection{Full-skin defect}

For in vivo experiments, scaffolds were prepared as for the oxygen release measurements, but in this case Integra ${ }^{\circledR}$ dermal regeneration template (Integra Life Science Corporation, Plainsboro, NJ, USA) was used. All procedures on laboratory animals were approved by the District Government of Upper Bavaria (Regierung von Oberbayern) and performed according to the current German animal welfare act (TierSchG). Experiments were performed on female Skh1 hairless mice (Crl:SKH1-Hr ${ }^{h r}$, Charles River, Sulzfeld, Germany) of 6-8 weeks age and body weight of 20-25 g. Under inhalative anesthesia (Isoflurane, Baxter Germany, Unterschleissheim, Germany) a bilateral $10 \mathrm{~mm}$ full skin defect was created on the back of the mice by first using a $10 \mathrm{~mm}$ biopsy punch to delineate the excision areas, and then, using fine surgical scissors to remove the skin. Next, a round piece of titanized mesh (ø $13 \mathrm{~mm}$ ) was placed over the wound bed and under the wound edges. Control and microalgae-seeded scaffolds were placed over the mesh and then sutured to the adjacent wound edges with 6-8 single knots, leaving the edges slightly over the scaffold. The wounds were covered with a transparent dressing (V.A.C. ${ }^{\circledR}$ Drape, KCI Medical Products, Wimborne Dorset, UK), which was also sutured to the skin. In order to trigger the oxygen production, cages were equipped with a flexible LED module $(20 \mathrm{~V}, 14.4 \mathrm{~W}$, WW, $2700 \mathrm{~K}$, Ledxon, Landshut, Germany) to stimulate the photosynthetic scaffolds with additional 3500 Lux during daytime. According to the TierSchG, animals must be subjected to circadian cycles of $12 / 12 \mathrm{~h}$ light/darkness. Thus, photosynthetic implants could not be exposed for more than $12 \mathrm{~h}$ to light stimulation per day. Control animals were kept under the same light stimulation conditions. At the end of the experimental times, animals were sacrificed via isofluorane overdose and the skin from the back including the scaffolds was excised for further analysis. To evaluate the systemic effects of implanting photosynthetic biomaterial, weight loss of the animals at the end of the experimental time was recorded. Further, the immune organs, thymus, spleen, mesenteric (MLN), and iliac lymph nodes (LN), were removed and their size and weight were measured and related to their final body weight. Whole blood was collected directly from the heart and allowed to clot by leaving it undisturbed on ice for $1 \mathrm{~h}$. The clot was removed by centrifugation at $2000 \mathrm{~g}$ for $10 \mathrm{~min}$ at $4{ }^{\circ} \mathrm{C}$. Serum was transferred into clean test tubes and stored at $-80^{\circ} \mathrm{C}$ for further analysis. Three animals per group were used in this study.

\subsection{Fluorescence-activated cell sorting (FACS)}

Harvested organs were homogenized by mechanical disruption and filtered through a $100 \mu \mathrm{m}$ cell strainer. Following red blood cell lysis treatment, $1 \cdot 10^{6}$ cells were stained for flow cytometry using the following fluorochrome conjugated antibodies: anti-CD4 (RM45, Ordering No: 12004183), anti-CD19 (1D3, Ordering No: 12019381), anti-CD69 (H1.2F3, Ordering No: 45069182), anti-CD44 (IM7, Ordering No: 11-0441-81) all from eBioscience (eBioscience, Inc., CA, USA), and anti-CD8a (53-6.7, Ordering No: 553033) from BD Pharmingen (BD Pharmingen, NJ, USA). Samples were acquired using FACS CantoII (BD Biosciences, NJ, USA) and analyzed with FlowJo software (Treestar, Or, USA). 


\subsection{Cytokine beads assay}

The concentration of inflammatory cytokines in serum was determined by a BD ${ }^{\text {тм }}$ CBA Mouse Inflammation Kit (BD Pharmingen, NJ, USA) following the manufacturer's instructions. This assay quantitatively measures IL-6, IL-10, IL-12p70, MCP-1, IFN- $\gamma$, and TNF protein levels in a single sample. Briefly, capture beads conjugated with a specific antibody and detector phycoerythrin (PE)conjugated antibodies are incubated with an unknown sample. Then, sandwich complexes of capture bead, analyte and detection reagent are formed, and provide a fluorescent signal which is measured by flow cytometry. This signal is compared to a calibration curve obtained with recombinant cytokines in order to obtain the amount of bound analyte.

\subsection{Viability of the algae ex vivo}

Biopsies of the explanted scaffolds of approximately $12 \mathrm{~mm} \times 1 \mathrm{~mm}$ were taken after 7 and 14 days post-implantation (dpi). Then, they were incubated for $24 \mathrm{~h}$ in liquid TAPS medium with $10 \mu \mathrm{g} / \mathrm{ml}$ paromomycin (Sigma-Aldrich, MO, USA), centrifuged $(290 \times \mathrm{g}, 5 \mathrm{~min})$ and cultured for two weeks in TAPS-agar plates containing the same concentration of antibiotics.

\subsection{Visualization and quantification of the vascular network}

In order to quantify the rate of vascularization over the wound, tissue transillumination and digital segmentation was performed as described before [21]. Briefly, the skin from the back of the animals was removed and stretched out on a petri dish over a transilluminator device. Then, pictures of the growing vascular network over the wound bed were taken using a stereomicroscope (Stereomicroscope Stemi 2000-C, Carl Zeiss AG, Oberkochen, Germany). A mask of the growing network under each scaffold and the vasculature of the uninjured skin was drawn by hand and then processed by the VesSeg-Tool analysis software [22]. For the analysis, the number and length of vessels and not their size was regarded. Therefore, prior to the estimation of the area covered by the microvasculature, all vessels were thinned to a width of one pixel. Results were expressed as percentage of vascularized area compared to normal skin.

\subsection{Histology and immunohistochemistry}

Sections $(2 \mu \mathrm{m})$ of diverse organs and the explanted tissues (fixed in $4 \%$ paraformaldehyde and paraffin-embedded) were stained with Hematoxylin (Ordering No: 351945S, VWR Chemicals, Radnor, PA, USA)/Eosin (1B-425, Waldeck GmbH \& Co. KG, Münster, Germany) or various antibodies. Incubation was performed with Bond Polymer Refine Detection Kit (Ordering No: DS9800, Leica Biosystems, Nussloch, Germany) on a Bond MAX (Leica). For quantification of the stainings, slides were scanned using a SCN400 slide scanner (Leica Biosystems, Nussloch, Germany) and analyzed using Tissue IA image analysis software (Slidepath, Leica Biosystems, Nussloch, Germany). Antibodies raised against the following antigens were used for immunohistochemistry: CD31 (1:40; Ordering No: DIA310, Dianova GmbH, Hamburg, Germany), aSMA (1:5000; Ordering No: A2547, Sigma-Aldrich, MO, USA), PDGFRb (1:50; Ordering No: 3169, CellSignalling, MA, USA), B220 (1:3000; Ordering No: 553084, BD Biosciences, NJ, USA), CD3 (1:250; Ordering No: RBK024, Zytomed, Berlin, Germany), F4/80 (1:120; Ordering No: T-2006, BMA Biomedicals AG, Augst Switzerland), Ly6G (1:600; Ordering No: 551459, BD Pharmingen, NJ, USA). Digital analysis and quantification of positively stained cells were performed with the software Image [18].

\subsection{Statistical analysis}

All assays were repeated in at least 3 independent experiments. If not stated otherwise, data was expressed as mean \pm SD. Twotailed Student's t-test was used to compare differences between 2 groups. Differences among means were considered significant when $\mathrm{p} \leq 0.05$.

\section{Results}

\subsection{Engraftment of photosynthetic scaffolds does not trigger a significant immune response}

In our previous work, we showed that, after short periods of implantation, the use of scaffolds seeded with the microalgae C. reinhardtii, did not trigger a significant inflammatory response in an athymic immunodeficient nude mice model [14]. Here, as a further step to evaluate the safety of HULK in vivo, photosynthetic scaffolds for dermal regeneration were used to replace full-skin defects in fully immunocompetent mice [23] and the results were evaluated after fourteen days. No complications were observed during or after the surgical procedure, and no significant differences in weight were detected among the experimental groups (Control $97.1 \% \pm 5.5 \%$, C.r. $103.8 \% \pm 5.0 \%$ ). Moreover, secondary lymphoid organs (e.g. spleen, thymus, mesenteric and iliac lymph nodes) did not show any significant increase in size nor signs of inflammation (Fig. 1A, B). Histological analysis of these organs (e.g. splenic white pulp follicles, B-cells follicles in MLNs) revealed normal micro-architectural organization, cell composition and, did not display any macroscopic or histological signs of possible immune activation (Fig. 1C, Supplementary Fig. S1). Besides, we could not detect any adverse effects in non-lymphoid organs (e.g. kidney, liver, lung, pancreas (Supplementary Fig. S1), similarly suggesting the lack of immune reaction. Furthermore, leukocyte activation did not vary between the experimental and control groups (Fig. 1D) and no differences in the serum levels of inflammation-related cytokines were detected (Fig. 1E). These results suggest that photosynthetic scaffolds can be implanted in an immunocompetent host without causing a major inflammation or triggering a significant immune response.

\subsection{C. reinhardtii microalgae can be genetically modified to secrete bioactive recombinant human growth factors}

In order to go further in the development of autotrophic engineered tissues, we next explored the idea of engineering biomaterials that, in addition to oxygen, could locally deliver other pro-regenerative molecules in situ. As a proof of concept, in this work we created a genetically modified strain of $C$. reinhardtii, capable to synthesize and secrete the angiogenic human growth factor VEGF.

For genetic modification, the human sequence encoding for the 165 amino acid polypeptide chain of the growth factor VEGF-A was codon-adapted for its expression in $C$. reinhardtii (Supplementary Fig. S2A). Upon transformation, the correct insertion of the transgene was evaluated first by southern blot, which showed the integration of a single copy of VEGF in the algae nuclear genome (Supplementary Fig. 2B). Then, we confirmed the correct size of the integrated human transgene using transgene-targeted PCR primers (Fig. 2A). Western blot analysis, showed that the recombinant growth factor had similar molecular weight as compared to a commercially available recombinant human VEGF monomer (Fig. 2B, $22.3 \mathrm{kD}$ versus $19.2 \mathrm{kD}$ ), given that the recombinant protein synthesized by the microalgae was preceded by the 21 amino acid export sequence of ARS2 $(1.97 \mathrm{kD})$. The production of the 
A

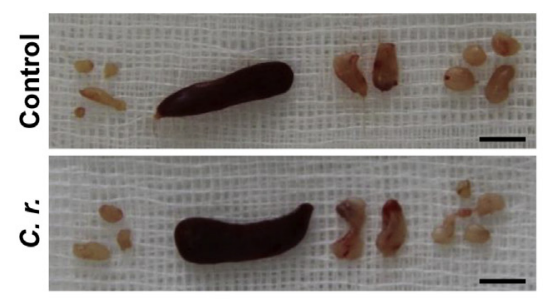

B

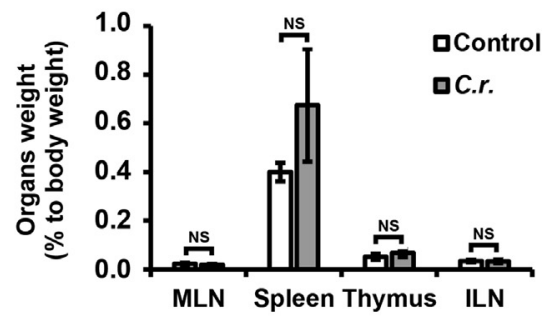

C
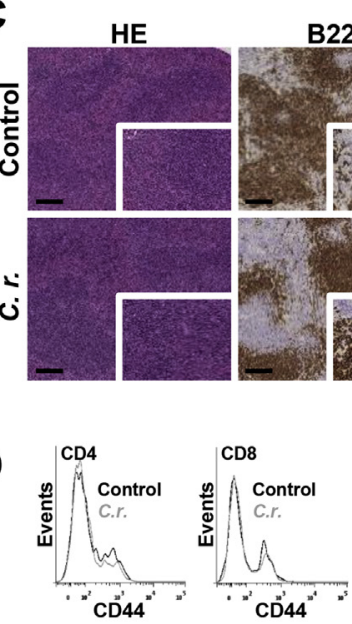

B220
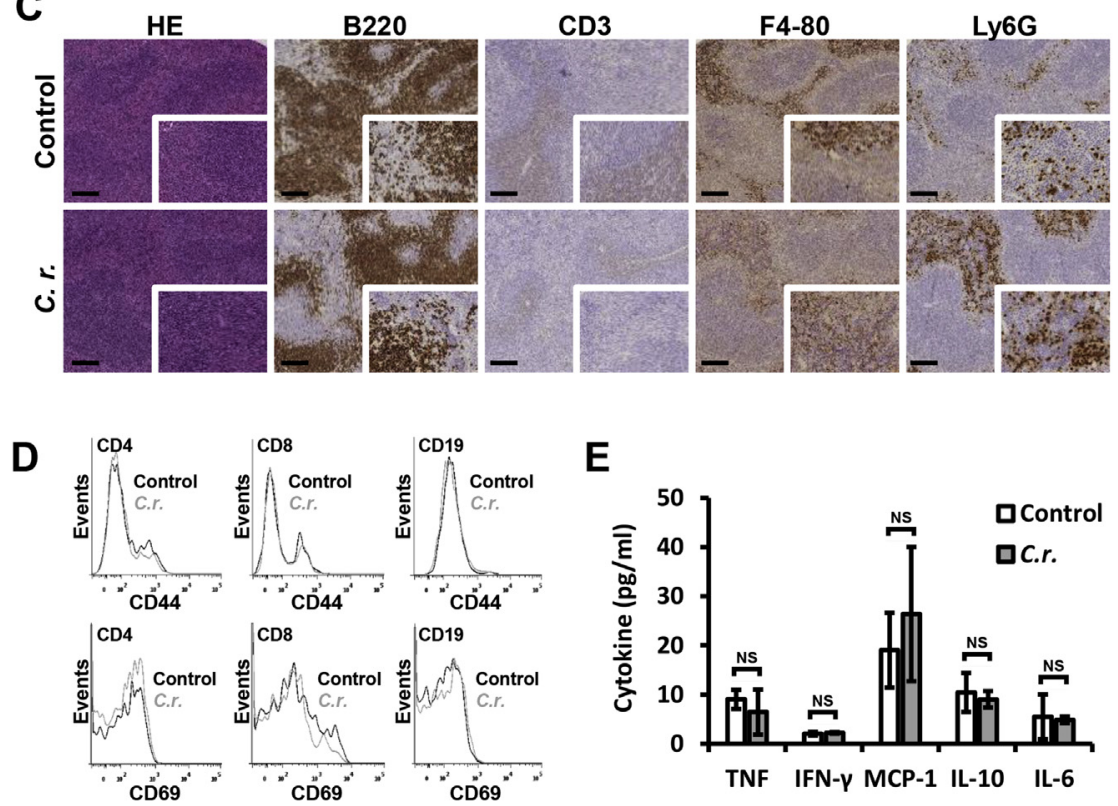

E

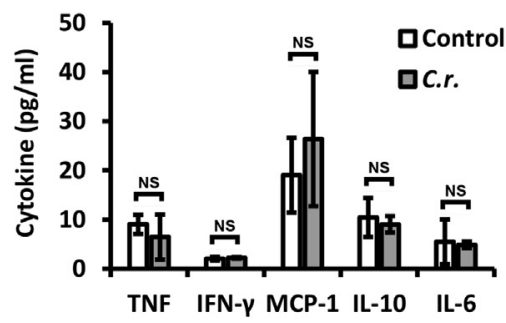

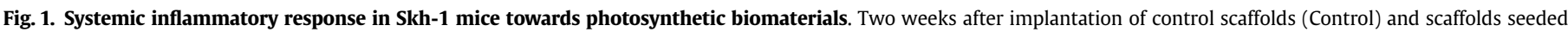

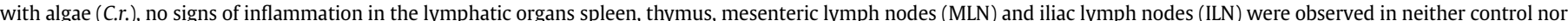

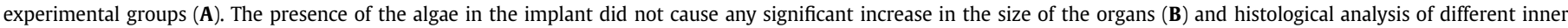

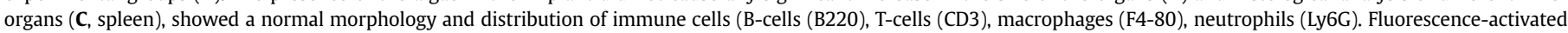

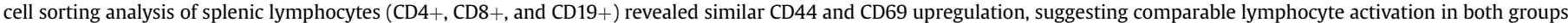

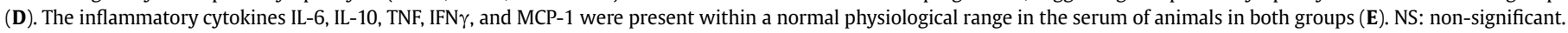
$\mathrm{N} \geq 3$. Scale bar represents $0.5 \mathrm{~cm}$ in $\mathbf{A}$ and $100 \mu \mathrm{m}$ in $\mathbf{C}$.

recombinant growth factor was quantitatively estimated by ELISA, and showed a secretion rate of $1.4 \pm 0.2 \mathrm{fg} \mathrm{VEGF} /$ cell $(28.0 \pm 4.38 \mathrm{ng}$ $\mathrm{VEGF} / \mathrm{ml}$ ) into the culture medium.

In order to evaluate the bioactivity of the recombinant VEGF, in vitro and in vivo experiments were performed. First, human primary endothelial cells were stimulated with culture medium taken from modified algae. The ligand-binding induced autophosphorylation of the VEGF-receptor 2 was confirmed by western-blot (Fig. 2C, D). Moreover, the activation of the VEGFsignaling pathway significantly increased the viability and migration capacity of the endothelial cells in vitro (Fig 2E, F). The bioactivity of the gene modified algae was tested in vivo using zebrafish larvae. For this, the microalgae were injected into the yolk sack of the embryos at one day post-fertilization, and three days later embryos were examined for morphological alterations in the vasculature. Whereas mock-injected fish or fish injected with wildtype algae did not show any alteration, an ingrowth of blood vessels towards the modified algae was evident in about $30 \%$ of the animals (Fig. 3), proving the bioactivity of the modified algae in vivo. Such vascular ingrowth was only observed when the majority of the algae remained in a ventro-central position within the yolk sack.

\subsection{Photosynthetic scaffolds are able to release recombinant} growth factors, in addition to oxygen, and promote tissue regeneration

In a next step, gene modified algae were seeded in clinically available scaffolds for dermal regeneration and the sustained release of photosynthetic oxygen and recombinant VEGF was evaluated in vitro. Similar to what we have previously described [14], when seeded at low concentrations, modified C. reinhardtii cells were able to proliferate within the biomaterial (Fig. 4A, B) and released substantial amounts of oxygen immediately upon light stimulation, reaching the saturation limit of the oxygen sensor after about $4 \mathrm{~h}\left(\mathrm{pO}_{2} \geq 50 \% ; 18.0 \mathrm{mg} / \mathrm{L}\right)$. In contrast, oxygen concentrations of non-photosynthetic control scaffolds steadily declined over the time (Fig. 4C). In addition, scaffolds seeded with gene modified algae sustained a constant release of recombinant VEGF for at least fourteen days in vitro (Fig. 4D). An average of $7.46 \pm 1.66 \mathrm{ng}$ VEGF/ $\mathrm{ml}$ was determined in the plateau of the secretion curve.

Finally, we studied the efficacy for the use of photosynthetic scaffolds in a dermal regeneration model in vivo, and evaluated their effects on the regeneration process and their capacity to 

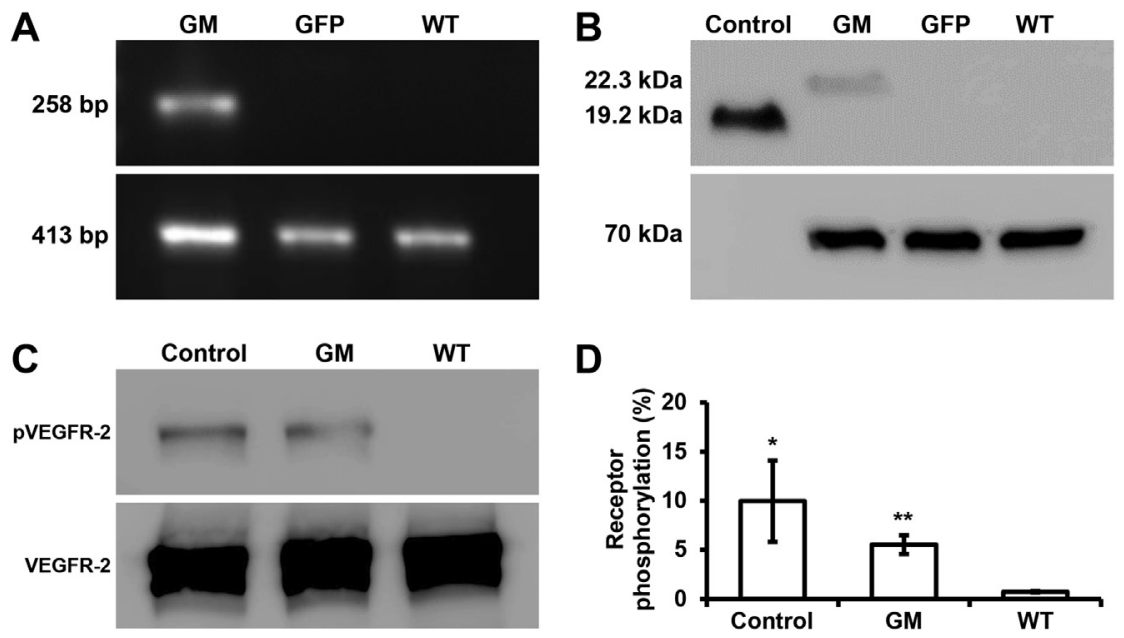

D
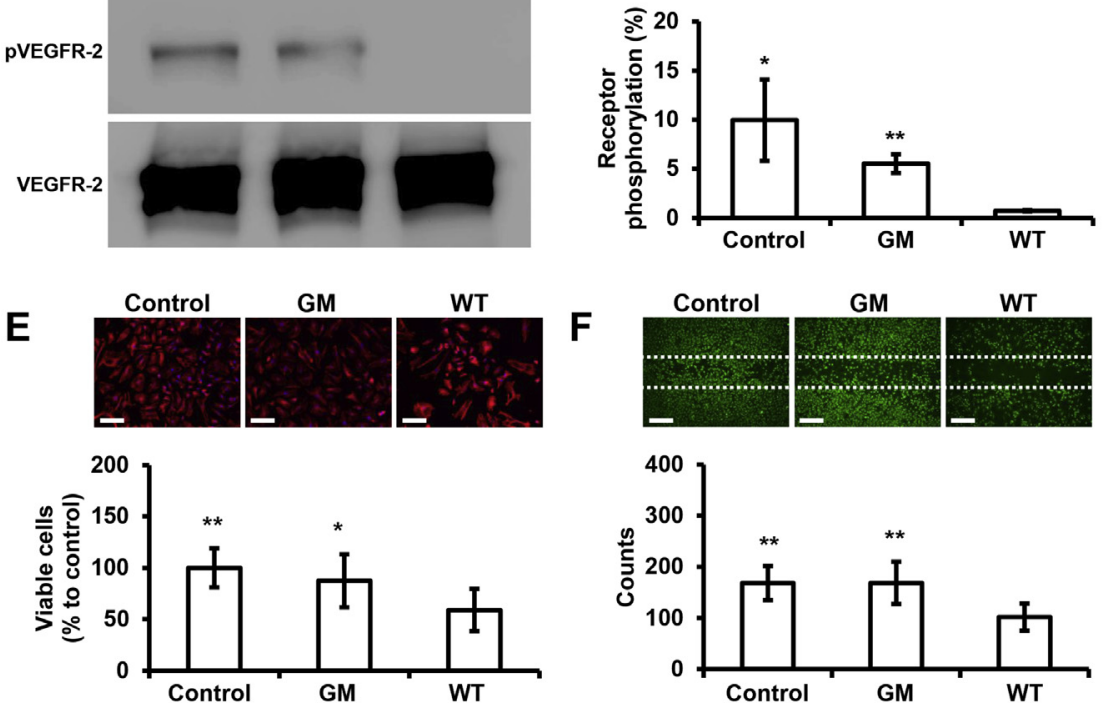

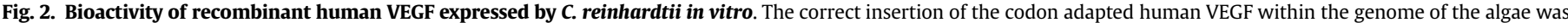

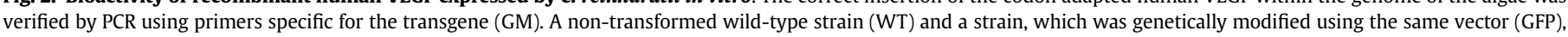

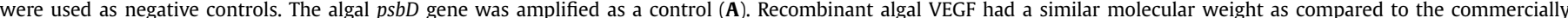

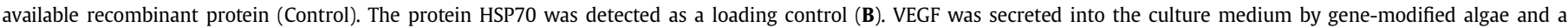

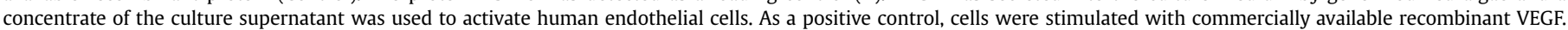

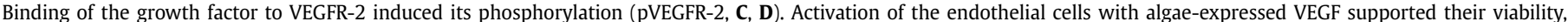

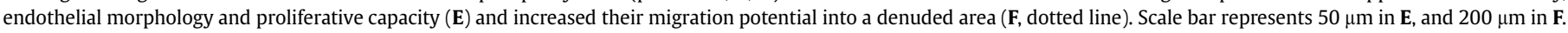
$\mathrm{N} \geq 3,{ }^{*} \mathrm{p}<0.05,{ }^{* *} \mathrm{p}<0.005$. Results are shown as mean \pm SEM in $\mathbf{E}$ and as mean \pm S.D in all other experiments.
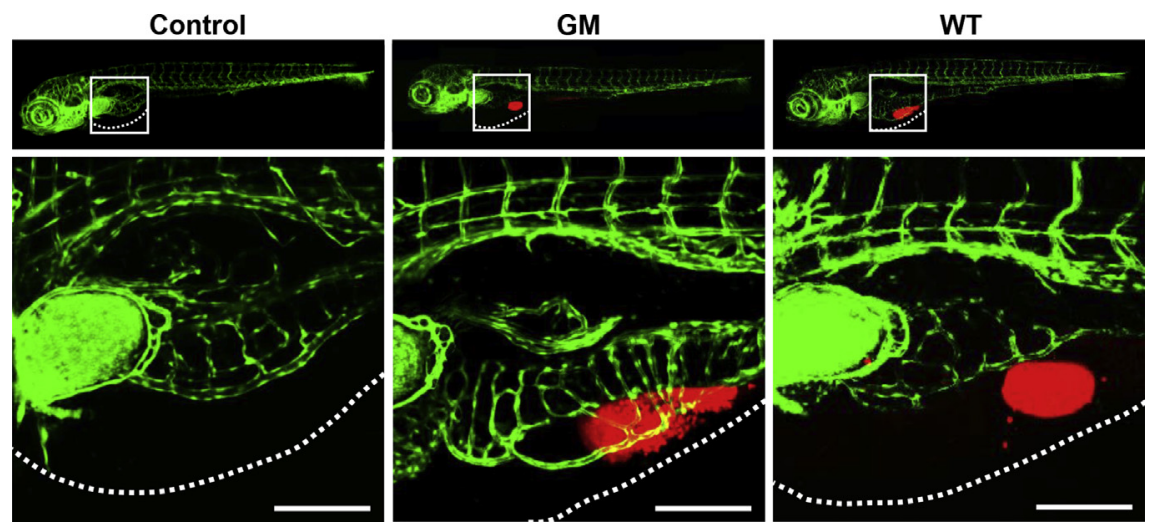

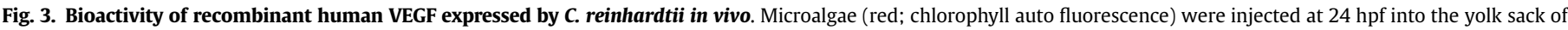

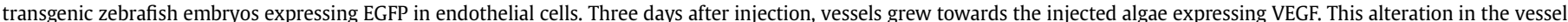

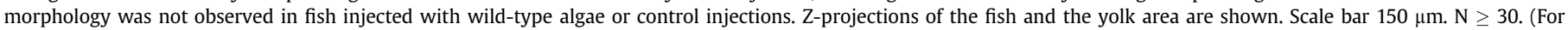
interpretation of the references to colour in this figure legend, the reader is referred to the web version of this article.)

deliver recombinant VEGF in situ. Here three groups of engrafted mice were compared: Control (non-seeded scaffolds), photosynthetic scaffolds (seeded with wild-type algae) and gene modified photosynthetic scaffolds (seeded with transgenic algae). Seven and fourteen days after engraftment, the general outcome of the scaffolds was macroscopically evaluated and no signs of local infection, inflammation, rejection or necrosis were observed in the surrounding wound area or within the engrafted scaffolds in either group. Additionally, no significant wound contraction was observed and the defect remained similar in size in all three groups (Fig. 5A). In order to determine the capacity of the modified algae to deliver the desired recombinant proteins in vivo, the amount of human 
A

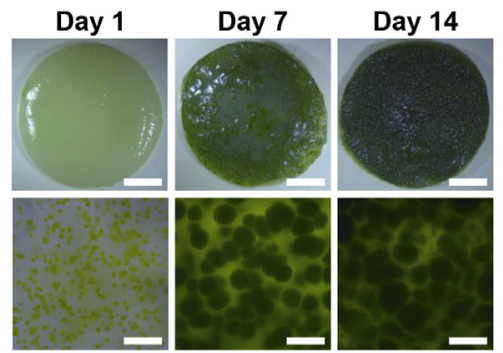

C

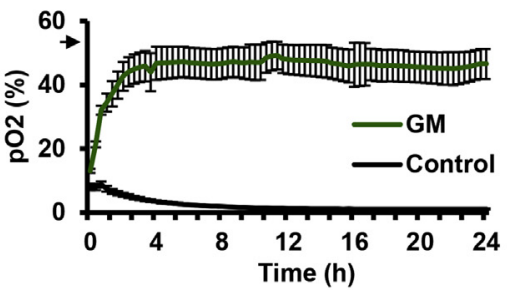

B
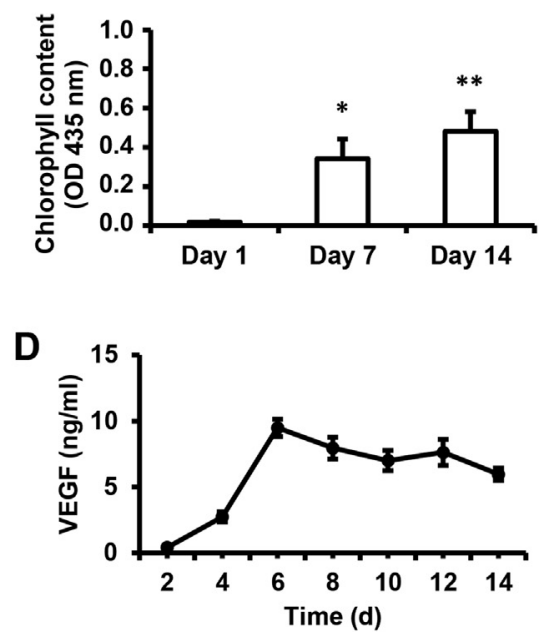

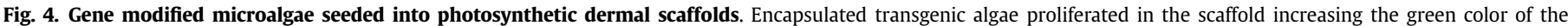

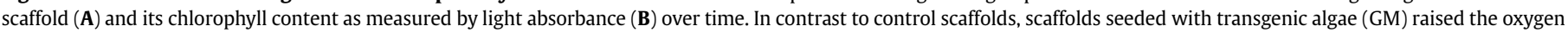

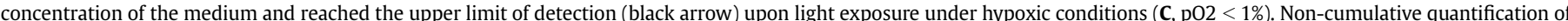

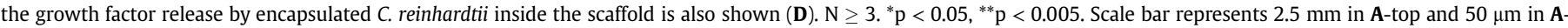
bottom. (For interpretation of the references to colour in this figure legend, the reader is referred to the web version of this article.)
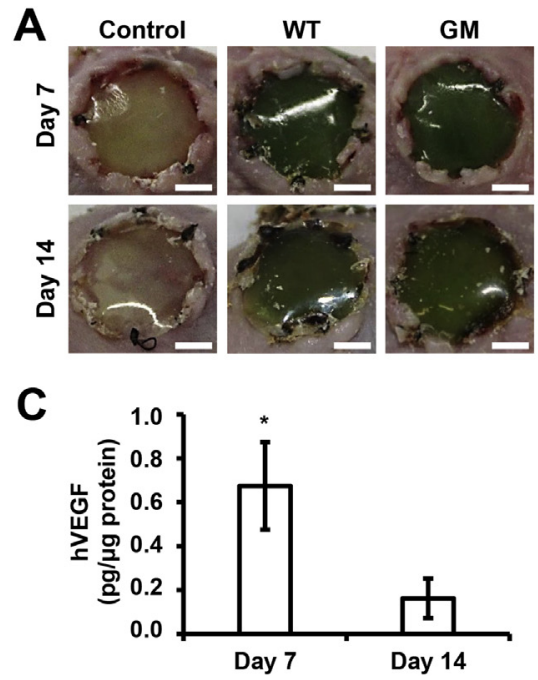

B

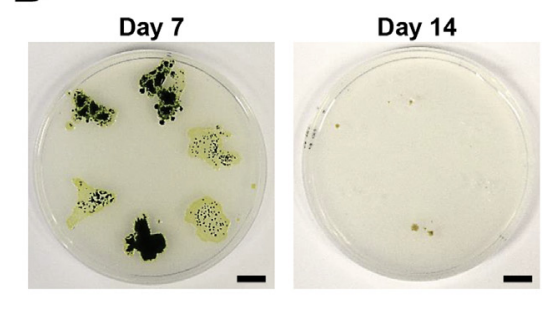

D

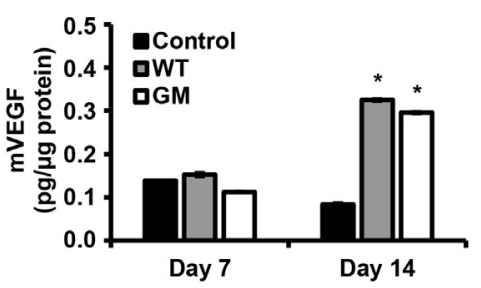

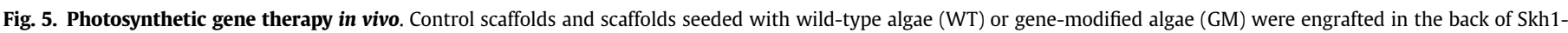

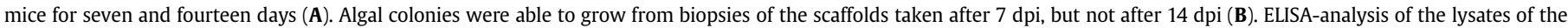

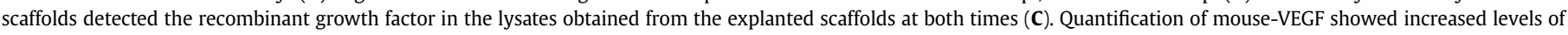
the endogenous factor in both groups with photosynthetic scaffolds after $14 \mathrm{dpi}(\mathbf{D})$. N $\geq 6 .{ }^{*} \mathrm{p}<0.05$. Scale bar represents $2.5 \mathrm{~mm}$ in $\mathbf{A}$ and $1 \mathrm{~cm}$ in $\mathbf{B}$.

VEGF in the wound was quantified at seven and fourteen days posttransplantation. Although, the recombinant molecule was detected at both experimental times, it was significantly lower at day fourteen (Fig. 5C). This decrease in the concentration was consistent with the survival curve of the algae in the wound, which was corroborated by the capacity of transgenic algae to regrow from biopsies of the explanted scaffolds after seven but not fourteen days post-implantation (Fig. 5B). Interestingly, after fourteen days, the sole presence of the algae promoted a significant increase of the endogenous mouse VEGF in both photosynthetic groups compared to the control (Fig. 5D).

After harvesting, implanted materials showed an ingrowth of vasculature from the wound edge towards the wound bed and below all three different implants (Fig. 6A). Unexpectedly, the presence of gene modified algae in the wound had only mild effects in promoting blood vessel formation, as seen after one week by an increased number of $\mathrm{CD} 31^{+}$cells in the wound (Fig. 6C, D). Yet, by day fourteen, the presence of the recombinant human VEGF did not significantly increase the vascularization rate of the scaffold (Fig. 6B). On the other hand, the implantation of photosynthetic scaffolds, seeded either with wild-type or transgenic algae, significantly enhanced the recruitment of endothelial cells (CD31 positive cells, Fig. 6C, D) and the formation of alpha smooth actin (aSMA) positive vessels in the wound bed area after fourteen days (Fig. 6E, F). Furthermore, photosynthetic scaffolds remarkably stimulated the formation of an extended granular-like tissue layer underneath, which covered the entire defect after fourteen days (Fig. 7A, C). When compared to the control scaffolds, this tissue 

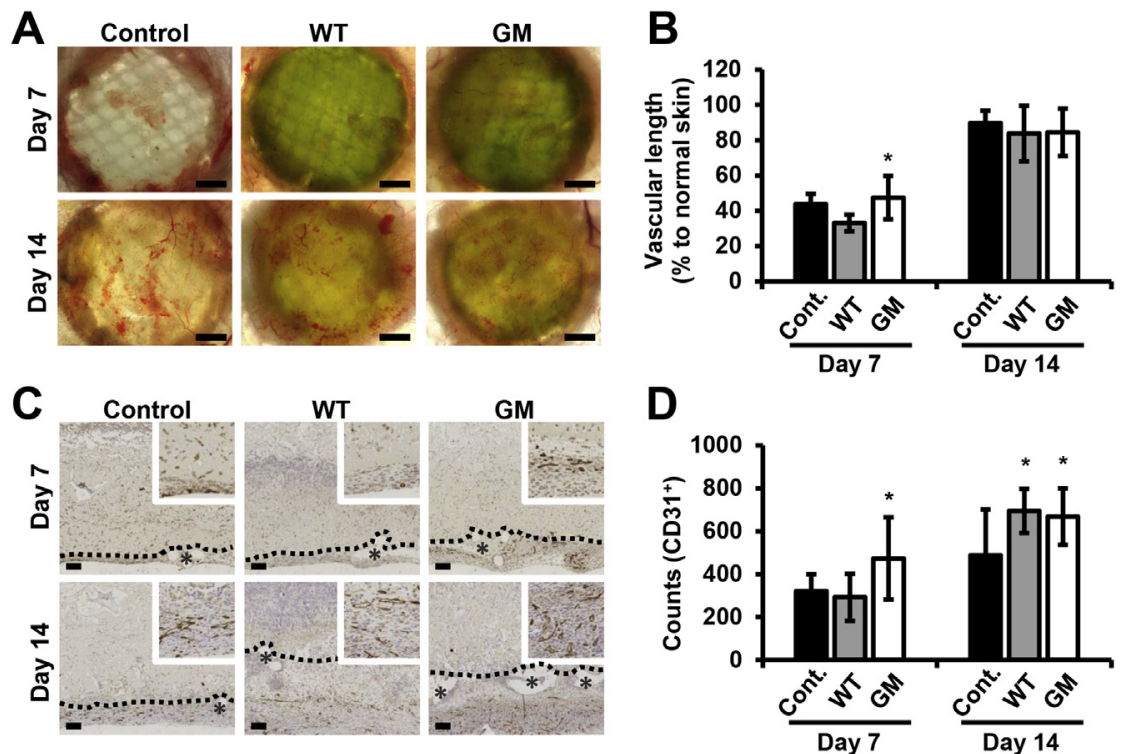

D
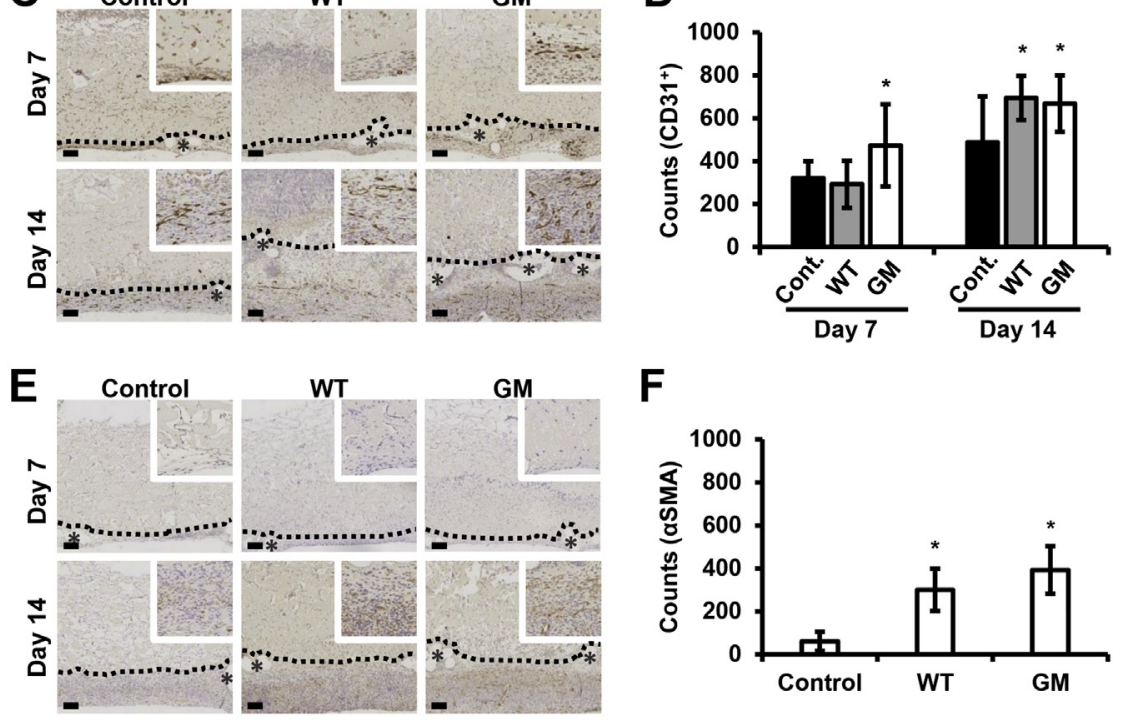

$\mathbf{F}$

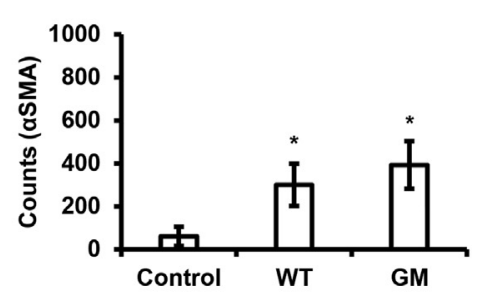

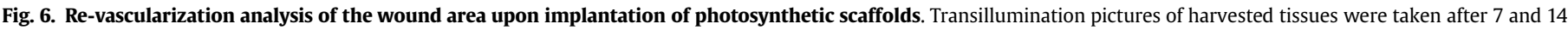

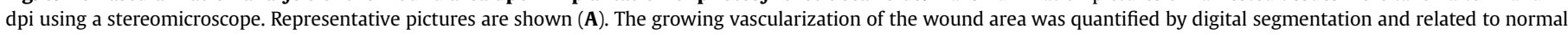

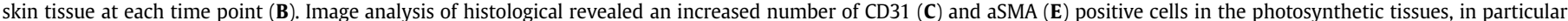

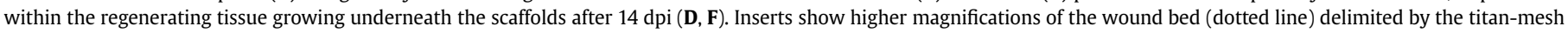
placed below the scaffold $\left({ }^{*}\right) . \mathrm{N} \geq 6{ }^{*} \mathrm{p}<0.05$. Scale bar represents $2.5 \mathrm{~mm}$ in $\mathbf{A}$, and $100 \mu \mathrm{m}$ in $\mathbf{C}$ and $\mathbf{E}$.

produced a firm bond to the subcutaneous muscle as well as to the dermis of the wound edge and was significantly thicker at both the wound bed and below the wound edge (Fig. 7B, D). Additionally, it was more densely populated with cells and a more pronounced extension of the epidermis over the wound edge was observed in both photosynthetic scaffolds, when compared to the control group (Fig. 7C). Interestingly, a higher rate of matrix degradation was observed in the photosynthetic scaffolds as judged by the loss of the scaffold's original structure at day fourteen (Fig. 7A), and the brittleness of the photosynthetic scaffolds noticed during the preparation of the histological samples (data not shown).

\section{Discussion}

We previously proposed that the development of a photosynthetic biomaterial, capable of oxygen self-production, could offer an unlimited local source of oxygen supply for the regenerating tissue, which would merely depend on light stimulation [13]. In this approach, the green unicellular microalgae $C$. reinhardtii are incorporated into scaffolds and exposed to illumination to trigger photosynthesis and achieve oxygen production in situ. Moreover, we also demonstrated that $C$. reinhardtii microalgae did not induce a significant inflammatory response in two different species [14]. However, those previous results mainly evaluated the interaction of the innate immune system with the algae. In order to further asses the biocompatibility of the photosynthetic biomaterials, in this study, photosynthetic scaffolds for dermal regeneration were engrafted in the back of fully immunocompetent mice. Under this new experimental setting, no overt immune response was observed in the host organism (Fig. 1), and in agreement with our previous study [14], these results further support the safety of the HULK-approach within the context of tissue engineering.

In a further step towards the development of fully autotrophic tissues, we made use of genetic manipulation tools to engineer a scaffold that, in addition to oxygen, would be able to provide other therapeutic molecules. Since re-establishment of a functional vascular network is believed to be key for successful wound repair $[24,25]$, in this work we decided to release the human growth factor VEGF to introduce the concept of photosynthetic gene therapy.

Compared to other eukaryotic cells, the use of microalgae as a vehicle could have an important impact on gene therapy, primarily because their survival does not depend on external oxygen supply. Besides, in this approach human transgenes are expressed from the algal genome, which translates into no risk of insertional mutagenesis, or potential oncogenicity for the human host. In this regard, $C$. reinhardtii represents an attractive organism for recombinant protein expression, because it combines the fast and easy growth of unicellular microorganisms, with the advantages of eukaryotic protein expression. Particularly, transgene expression from the nuclear genome bids to direct proteins to organelles of the secretory pathway, thus enabling post-translational modifications and their final targeting for excretion [26], in contrast to chloroplast expression [27]. Hence, today the list of recombinant proteins successfully expressed by $C$. reinhardtii includes stable active 
A

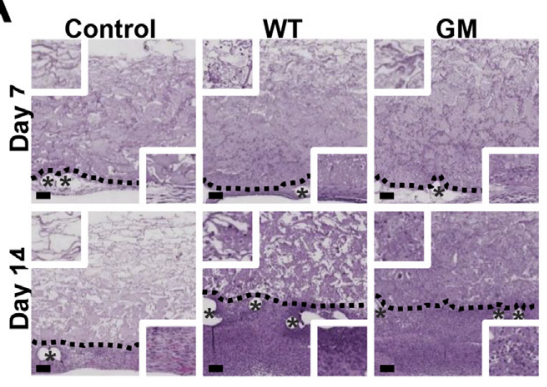

C

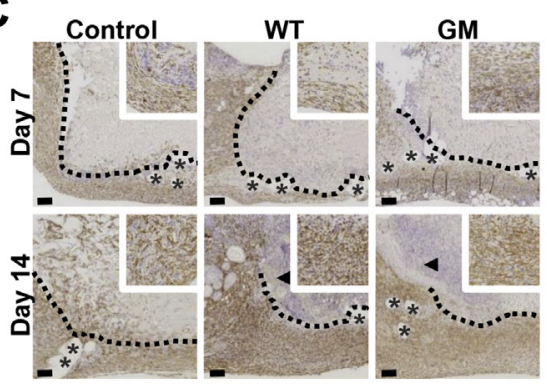

B

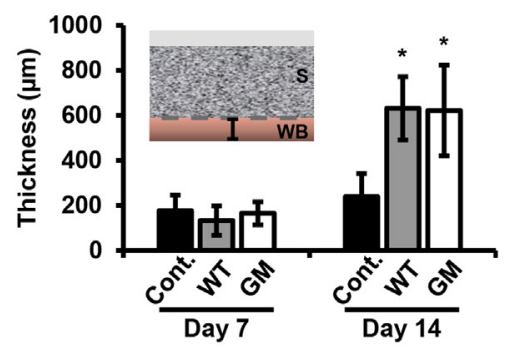

D

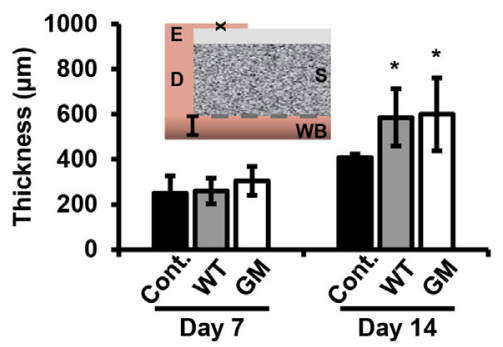

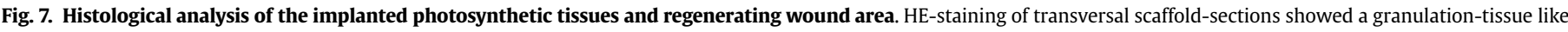

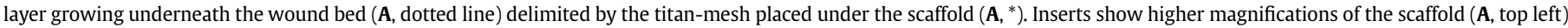

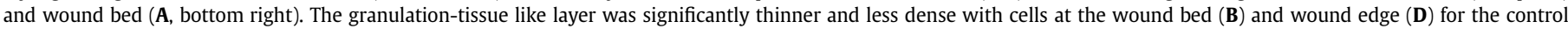

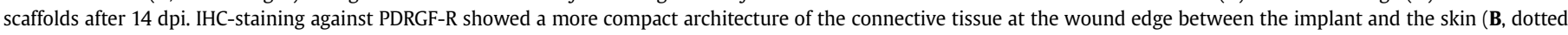

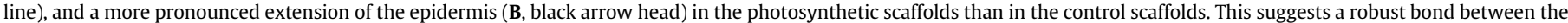

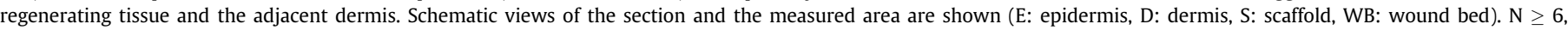
${ }^{*} \mathrm{p}<0.05$. Scale bar represents $100 \mu \mathrm{m}$.

monoclonal antibodies, antigenic proteins for oral vaccines, and human growth factors [28].

The recombinant growth factor secretion by $C$. reinhardtii into the culture medium, was found within the range of VEGF concentrations that are known to stimulate angiogenesis in vitro, and have been previously used in other tissue engineered approaches $[29,30]$, although the expression rates of the algae are lower to those accomplished using retrovirally transfected murine myoblasts [31,32]. Nevertheless, the stimulation of human cells with the recombinant growth factor induced the autophosphorylation of the VEGFR-2 receptor and had direct consequences in endothelial cell survival, migration and proliferation in vitro (Fig. 2C-F). Further, the injection of the transgene algae into zebrafish (Fig. 3), induced similar alterations on the developing vascular architecture to those described upon injection of other angiogenic molecules or angiogenesis promoting cells [33-35]. Based on these results we conclude that $C$. reinhardtii microalgae are capable to synthesize and secrete functional pro-angiogenic growth factors in physiologically relevant amounts.

When seeded in a dermal scaffold, the transgenic algae sustained a constant release of both oxygen and growth factors for prolonged periods of time (Fig. 4C, D). As shown before [14], the biocompatible fibrin encapsulation of $C$. reinhardtii grants a homogeneous confinement of the algae inside the scaffolds and at the same time, allows the diffusion of oxygen produced by the proliferating algae through the scaffold (Fig. 4 A, B). In this study, we were also able to show a similar release of VEGF, and the averaged growth factor concentration was found in the range of previously described tissue engineering approaches [36-38]. Yet, in contrast to the release kinetics of some of them, there is no peak of expression or an early secretion burst. The expression curve of the photosynthetic scaffolds rather follows the growth curve of the seeded algae, resulting in a constant growth factor secretion sustained for as long as the algae are able to survive within the scaffold (Fig. 4D).

Upon engraftment, the recombinant VEGF was found in the protein lysates obtained from the explanted scaffolds after seven and fourteen days post-implantation (Fig. 5C). Since the efficient release of the recombinant growth factor from the biomaterial was demonstrated in vitro (Fig. 4D), we conclude that the transgenic algae were also able to synthesize VEGF in vivo, raising the concentration of the therapeutic molecule significantly in the wound area inside the implant. This demonstrates that genetically engineered $C$. reinhardtii could be used as an alternative method of growth factor delivery for tissue engineering purposes.

However, there was a significant net decrease on the measured concentrations of the growth factor after two weeks postimplantation. In this work, we showed that the algae remained alive and were able to regrow ex vivo after seven days of being implanted in the host, yet the viability of the algae seemed to terminate soon after this period of time (Fig. 5B). We therefore concluded that the amount of recombinant growth factor is in direct correlation with the number of viable microalgae in the photosynthetic biomaterial. This could represent an advantage in wound healing approaches, where transient delivery is desired. However, for other long-term applications, the use of different algae species with higher viability potential, or preconditioning strategies such as temperature [40] and light-dark cycles [41] could be explored to increase the viability of the algae in vivo. Moreover, conditions upon implantation could be further optimized. For instance, in the setting presented here, due to ethical concerns, scaffolds were exposed to only $12 \mathrm{~h}$ of light per day, which most probably affected key features such as survival and metabolic rates of the algae.

While the recombinant VEGF promoted endothelial cell migration and blood vessel formation after one week, the presence of the 
transgenic algae did not significantly increase the rate of vascularization over the wound bed afterwards (Fig. 6A-D). On this subject, it is has been demonstrated and described that the formation and maturation of functional blood vessels requires the coordinated action of additional soluble factors such as FGF, TGF $\beta$, PDGFB, Ang1, and PIGF [42-44]. Furthermore, it is known that timing is crucial in the orchestration of a complex process, such as wound re-vascularization. Although pro-angiogenic paracrine signals are present from day one after injury, the highest rate of angiogenesis takes place between days $7-14$ post-injury, following a peak in wound hypoxia at day $4[45,46]$. If compared with the viability of the algae in vivo, recombinant VEGF expression is not matching the requirements of the regenerating tissue and the availability of the growth factor decreases just when angiogenesis is starting.

In spite of this, the implantation of the photosynthetic scaffolds had two remarkable effects. First, a significant up-regulation of the endogenous mouse VEGF expression was found in the lysates obtained from the explanted photosynthetic scaffolds after two weeks post-implantation (Fig. 5D) and, as a most likely consequence, already the presence of the wild-type photosynthetic algae in the scaffold promoted the recruitment of endothelial progenitors (CD31 positive cells) and the formations of alpha smooth actin (aSMA) positive vessels in the wound bed area (Fig. 6C-F). In this regard, HBO-therapy has shown to increase angiogenesis in hypoxic and injured tissues suggestively via oxidant-mediated mechanisms and the up-regulation of angiogenesis-related molecules [47]. On the other hand, it is known that the primary angiogenesis-related gene activation mechanisms occur via activation of the hypoxia-sensor molecule HIF-1 $\alpha$ [48]. While algae respiration might be taking place during the dark periods to which the scaffolds are exposed, consuming therefore oxygen just as the animal cells, it has been also proven, that it is not the actual partial oxygen pressure, but rather the changes of it registered by the cells, what triggers the hypoxic pro-angiogenic transcriptional function of HIF- $1 \alpha$ [49]. Furthermore, the photosynthetic scaffolds stimulated the formation of an extended granulation-like tissue layer underneath the implanted scaffolds, which practically covered the defect two weeks after implantation (Fig. 7A-D). In this regard, it is known that epithelialization depends on the presence of a bed of healthy granulation tissue, which is in turn oxygen dependent [47]. All these results suggest that the presence of the algae in this setting is promoting tissue restoration, with the photosynthetic scaffold acting more like a bioreactor rather than a scaffold itself. If photosynthetic scaffolds could help maintaining an adequate tissue oxygenation, the risk of stalled wound healing or tissue loss due to chronic hypoxia could be prevented [50].

In this work, we showed that photosynthetic biomaterials seeded with gene modified algae can be used to deliver both oxygen and recombinant factors in situ. Most of the problems associated with foreign gene expression in the microalgae, such as inefficient DNA delivery, failing on the integration into the chromosome, inadequate recognition of the promoter region and epigenetic silencing mechanisms were successfully overcome due to the implemented strategy for recombinant protein expression that considered the biased codon usage, the necessary regulatory sequences and the required targeting signals for protein secretion. Following this system, further strains could be engineered to synthesize other therapeutic proteins such as antibiotics, enzymes, or immune-modulatory molecules, and used to create a proregenerative microenvironment in the wound area. In addition, by exploring the use of inducible promoters, gene expression by the microalgae could be coupled to the progress of wound healing and its requirements. Finally, because working with healthy young animals gives an elusive reference of the approach performance, further experiments should be performed to evaluate the therapeutic impact of photosynthetic scaffolds in larger tissue defects or models with impaired healing capacity.

\section{Conclusion}

In order to overcome hypoxia, we have previously suggested that photosynthetic tissue engineering could be used as an alternative source of oxygen supply to blood vessel-perfusion. In this work, we demonstrated that photosynthetic scaffolds can be compatibly engrafted in fully immunocompetent mice without causing a significant immune response and improving tissue regeneration. In addition, we were able to genetically engineer a $C$. reinhardtii strain to secrete the human growth factor VEGF, and deliver it into a wounded tissue in vivo. The results of this work represent a step forward in the development of autotrophic tissues, and suggest the use of engineered microalgae to treat a broad spectrum of hypoxic conditions as well as an alternative method for recombinant growth factor administration in other clinical set.

\section{Financial disclosure}

This work was partially financed by a CIRM-BMBF Early Translational II Award to J.T.E., ICGEB (CRP/CHI11-01) and the FONDAP Center for Genome regulation (Nr. 15090007) to both M.A. and J.T.E., and a DFG grant (Ni390/7-1) to J.N. M.H. was supported by an ERC starting grant (LiverCancerMech), the SFBTR 36 and the Stiftung für Bio-medizinische Forschung. The funders had no role in study design, data collection and analysis, decision to publish, or preparation of the manuscript.

\section{Competing interest}

JTE is founder and VP of Technology at SymbiOx Inc. This startup did not provide any financial support to this work but is closely related to some topics of this manuscript.

\section{Appendix A. Supplementary data}

Supplementary data related to this article can be found at http:// dx.doi.org/10.1016/j.biomaterials.2015.10.014.

\section{References}

[1] E. Bland, D. Dréau, K. Burg, Overcoming hypoxia to improve tissue-engineering approaches to regenerative medicine, J. Tissue Eng. Regen. Med. 7 (2013) 505-514.

[2] Lalan, Pomerantseva, Vacanti, Tissue engineering and its potential impact on surgery, World J. Surg. 25 (2001) 1458-1466.

[3] S. Werner, R. Grose, Regulation of wound healing by growth factors and cytokines, Physiol. Rev. 83 (2003) 835-870.

[4] S. Barrientos, O. Stojadinovic, M. Golinko, H. Brem, M. Tomic-Canic, Perspective Article: Growth factors and cytokines in wound healing, Wound Repair Regen. 16 (2008) 585-601.

[5] N. Greaves, K. Ashcroft, M. Baguneid, A. Bayat, Current understanding of molecular and cellular mechanisms in fibroplasia and angiogenesis during acute wound healing, J. Dermatol. Sci. 72 (2013) 206-217.

[6] M. Martino, S. Brkic, E. Bovo, M. Burger, D. Schaefer, T. Wolff, et al., Extracellular matrix and growth factor engineering for controlled angiogenesis in regenerative medicine, Front. Bioeng. Biotechnol. 3 (2015).

[7] K. Lee, E. Silva, D. Mooney, Growth factor delivery-based tissue engineering: general approaches and a review of recent developments, J. R. Soc. Interface 8 (2011) 153-170.

[8] S. Reed, B. Wu, Sustained growth factor delivery in tissue engineering applications, Ann. Biomed. Eng. 42 (2013) 1528-1536.

[9] T. Hirsch, M. Spielmann, F. Yao, E. Eriksson, Gene therapy in cutaneous wound healing, Front. Biosci. A J. Virtual Libr. 12 (2007) 2507-2518.

[10] K. Riedel, F. Riedel, U.R. Goessler, G. Holle, G. Germann, M. Sauerbier, Current status of genetic modulation of growth factors in wound repair, Int. J. Mol. Med 17 (2006) 183-193.

[11] S. Boeckle, E. Wagner, Optimizing targeted gene delivery: chemical 
modification of viral vectors and synthesis of artificial virus vector systems, AAPS J. 8 (2006) E731E742.

[12] F. Wegman, C. Öner, W. Dhert, J. Alblas, Non-viral gene therapy for bone tissue engineering, Biotechnol. Genet. Eng. Rev. 29 (2013) 206220.

[13] U. Hopfner, T.-L.L. Schenck, M.-N.N. Chávez, H.-G.G. Machens, A.-V.V. Bohne, J. Nickelsen, et al., Development of photosynthetic biomaterials for in vitro tissue engineering, Acta Biomater. 10 (2014) 2712-2717.

[14] T.L. Schenck, U. Hopfner, M.N. Chávez, H.-G.G. Machens, I. Somlai-Schweiger, R.E. Giunta, et al., Photosynthetic biomaterials: a pathway towards autotrophic tissue engineering, Acta Biomater. 15 (2015) 39-47.

[15] J. Neupert, D. Karcher, R. Bock, Generation of Chlamydomonas strains that efficiently express nuclear transgenes, Plant J. 57 (2009) 1140-1150.

[16] E. Harris, The Chlamydomonas Sourcebook: Introduction to Chlamydomonas and its Laboratory Use, second ed., Elsevier, Burlington, 2008.

[17] S.P. Crampton, J. Davis, C.C. Hughes, Isolation of human umbilical vein endothelial cells (HUVEC), J. Vis. Exp. JoVE 183 (2007).

[18] C.A. Schneider, W.S. Rasband, K.W. Eliceiri, NIH Image to ImageJ: 25 years of image analysis, Nat. Methods 9 (2012) 671-675.

[19] N. Lawson, B. Weinstein, Vivo imaging of embryonic vascular development using transgenic zebrafish, Dev. Biol. 248 (2002).

[20] P. Thévenaz, M. Unser, User-friendly semiautomated assembly of accurate image mosaics in microscopy, Microsc. Res. Tech. 70 (2007) 135-146.

[21] T.L. Schenck, M.N. Chávez, A.P. Condurache, U. Hopfner, F. Rezaeian, H.G. Machens, J.T. Egaña, A full skin defect model to evaluate vascularization of biomaterials in vivo, J. Vis. Exp 90 (2014), http://dx.doi.org/10.3791/51428.

[22] J. Egaña, A. Condurache, J. Lohmeyer, M. Kremer, B. Stöckelhuber, S. Lavandero, et al., Ex vivo method to visualize and quantify vascular networks in native and tissue engineered skin, Langenbeck's Arch. Surg. 394 (2008) 349-356.

[23] F. Benavides, T. Oberyszyn, A. VanBuskirk, V. Reeve, D. Kusewitt, The hairless mouse in skin research, J. Dermatol. Sci. 53 (2008).

[24] S. Eming, B. Brachvogel, T. Odorisio, M. Koch, Regulation of angiogenesis: wound healing as a model, Prog. Histochem. Cytochem. 42 (2007) 115-170.

[25] M.S. Wietecha, L.A. DiPietro, Therapeutic approaches to the regulation of wound angiogenesis, Adv. Wound Care (New Rochelle) 2 (2013) 81-86.

[26] A. Eichler-Stahlberg, W. Weisheit, O. Ruecker, M. Heitzer, Strategies to facilitate transgene expression in Chlamydomonas reinhardtii, Planta 229 (2009) 873-883.

[27] B. Rasala, M. Muto, P. Lee, M. Jager, R. Cardoso, C. Behnke, et al., Production of therapeutic proteins in algae, analysis of expression of seven human proteins in the chloroplast of Chlamydomonas reinhardtii, Plant Biotechnol. J. 8 (2010) 719-733.

[28] B. Rasala, S. Mayfield, Photosynthetic biomanufacturing in green algae; production of recombinant proteins for industrial, nutritional, and medical uses, Photosynth. Res. 123 (2014) 227-239.

[29] R. Elia, P.W. Fuegy, A. VanDelden, M.A. Firpo, G.D. Prestwich, R.A. Peattie, Stimulation of in vivo angiogenesis by in situ crosslinked, dual growth factorloaded, glycosaminoglycan hydrogels, Biomaterials 31 (2010) 4630-4638.

[30] K.T. Morin, R.T. Tranquillo, In vitro models of angiogenesis and vasculogenesis in fibrin gel, Exp. Cell Res. 319 (2013) 2409-2417.

[31] C. Ozawa, A. Banfi, N. Glazer, G. Thurston, M. Springer, P. Kraft, et al., Microenvironmental VEGF concentration, not total dose, determines a threshold between normal and aberrant angiogenesis, J. Clin. Investig. 113 (2004) $516-527$.

[32] A. Banfi, Degenfeld G. von, R. Gianni-Barrera, S. Reginato, M. Merchant, D. McDonald, et al., Therapeutic angiogenesis due to balanced single-vector delivery of VEGF and PDGF-BB, FASEB J. 26 (2012) 2486-2497.

[33] H. Habeck, J. Odenthal, B. Walderich, H. Maischein, S. Schulte-Merker, Analysis of a zebrafish VEGF receptor mutant reveals specific disruption of angiogenesis, Curr. Biol. ; CB 12 (2002) 1405-1412.

[34] S. Nicoli, G. Sena, M. Presta, Fibroblast growth factor 2-induced angiogenesis in zebrafish: the zebrafish yolk membrane (ZFYM) angiogenesis assay, J. Cell. Mol. Med. 13 (2009) 2061-2068.

[35] D. Li, X.P. Li, H.X. Wang, Q.Y. Shen, X.P. Li, L. Wen, X.J. Qin, Q.L. Jia, H.F. Kung, Y. Peng, VEGF induces angiogenesis in a zebrafish embryo glioma mode established by transplantation of human glioma cells, Oncol. Rep 28 (2012) 937-942.

[36] T. Richardson, M. Peters, A. Ennett, D. Mooney, Polymeric system for dual growth factor delivery, Nat. Biotechnol. 19 (2001) 1029-1034.

[37] A. Reckhenrich, U. Hopfner, F. Krötz, Z. Zhang, C. Koch, M. Kremer, et al. Bioactivation of dermal scaffolds with a non-viral copolymer-protected gene vector, Biomaterials 32 (2011) 19962003.

[38] O. Oliviero, M. Ventre, P. Netti, Functional porous hydrogels to study angiogenesis under the effect of controlled release of vascular endothelial growth factor, Acta Biomater. 8 (2012) 3294-3301.

[40] Tanaka, Nishiyama, Murata, Acclimation of the photosynthetic machinery to high temperature in Chlamydomonas reinhardtii requires synthesis de novo of proteins encoded by the nuclear and chloroplast genomes, Plant Physiol. 124 (2000) 441-449.

[41] J.D. Rochaix, Reprint of: Regulation of photosynthetic electron transport Biochim. Biophys. Acta 2011 (1807) 878-886.

[42] M.W. Laschke, A. Elitzsch, B. Vollmar, P. Vajkoczy, M.D. Menger, Combined inhibition of vascular endothelial growth factor (VEGF), fibroblast growth factor and platelet-derived growth factor, but not inhibition of VEGF alone effectively suppresses angiogenesis and vessel maturation in endometriotic lesions, Hum. Reprod. 21 (2006) 262-268.

[43] M. Potente, H. Gerhardt, P. Carmeliet, Basic and therapeutic aspects of angiogenesis, Cell 146 (2011).

[44] D. Ribatti, E. Crivellato, "Sprouting angiogenesis", a reappraisal, Dev. Biol. 372 (2012).

[45] Z. Haroon, J. Raleigh, C. Greenberg, M. Dewhirst, Early wound healing exhibits cytokine surge without evidence of hypoxia, Ann. Surg. 231 (2000) 137.

[46] A. Shaterian, A. Borboa, R. Sawada, T. Costantini, B. Potenza, R. Coimbra, et al. Real-time analysis of the kinetics of angiogenesis and vascular permeability in an animal model of wound healing, Burns 35 (2009) 811817.

[47] H. Hopf, M. Rollins, Wounds: an overview of the role of oxygen, Antioxid. Redox Signal. 9 (2007) 1183-1192.

[48] A. Tandara, T. Mustoe, Oxygen in wound healing-more than a Nutrient World J. Surg. 28 (2004) 294-300.

[49] C. Sen, Wound healing essentials: let there be oxygen, Wound Repair Regen. 17 (2009) 1-18.

[50] B. Krock, N. Skuli, C. Simon, Hypoxia-induced angiogenesis good and evil Genes Cancer 2 (2011) 1117-1133. 